\title{
Time course of regional brain activation associated with onset of auditory/verbal hallucinations
}

Ralph E. Hoffman, Adam W. Anderson, Maxine Varanko, John C. Gore and Michelle Hampson

\section{Summary}

The time course of brain activation prior to onset of auditory/ verbal hallucinations was characterised using functional magnetic resonance imaging in six dextral patients with schizophrenia. Composite maps of pre-hallucination periods revealed activation in the left anterior insula and in the right middle temporal gyrus, partially replicating two previous case reports, as well as deactivation in the anterior cingulate and parahippocampal gyri. These findings may reflect brain events that trigger or increase vulnerability to auditory/verbal hallucinations.

\section{Declaration of interest}

None. Funding detailed in Acknowledgements.
Auditory/verbal hallucinations of spoken speech, which occur in $60-80 \%$ of people with schizophrenia, are associated with high levels of distress and functional disability. Although neuroimaging studies have associated regional brain activation with such hallucinations, the data generally cannot differentiate activation reflecting their production from downstream consequences of these events such as shifts in arousal or registration of hallucinations in verbal memory. Two reports of time course of brain activation associated with auditory/verbal hallucinations characterised using functional magnetic resonance imaging (fMRI) are therefore of special interest. Lennox et al described activation in the right middle temporal gyrus detected $3 \mathrm{~s}$ prior to hallucination onset followed by bitemporal activation in one patient. ${ }^{1}$ Shergill et al detected left inferior frontal and right middle temporal gyral activation arising a full $6-9 \mathrm{~s}$ prior to hallucination onset in two patients. $^{2}$ The pre-hallucination timing of these activations suggests neural events triggering or increasing vulnerability to auditory/verbal hallucinations. We attempted to replicate and extend these reports by studying a larger patient sample.

\section{Method}

\section{Participants}

Six right-handed patients with either schizophrenia or schizoaffective disorder diagnosed using DSM-IV ${ }^{3}$ criteria were studied. These patients were treated with clozapine $(n=3)$, haloperidol $(n=1)$ or olanzapine $(n=1)$, or no antipsychotic drug $(n=1)$. Informed written consent was obtained from all participants prior to the study.

\section{Imaging}

We used a $1.5 \mathrm{~T}$ Signa LX scanner (General Electric, Milwaukee, Wisconsin, USA) to obtain $T_{1}$-weighted structural images of 14 contiguous $6 \mathrm{~mm}$ slices acquired parallel to the anterior-posterior commissural line. These images were used for anatomical identification and coincided with slices in the functional runs. For functional runs, participants were instructed to depress a button with their right hand to mark the onset of each auditory/ verbal hallucination and to release the button at termination of the event; six or seven of these runs lasting 4 min $6 \mathrm{~s}$ each were used for each participant. Functional runs - 14 axial-oblique slices of single-shot echoplanar imaging: repetition time (TR) $1500 \mathrm{~ms}$, echo time (TE) $60 \mathrm{~ms}$, flip angle $60^{\circ}, 64 \times 64$ acquisition matrix, voxel size $3.125 \mathrm{~mm} \times 3.125 \mathrm{~mm} \times 6 \mathrm{~mm}$ - were motion-corrected using the statistical parametric mapping algorithm and subjected to a spatial Gaussian filter with 2 pixels full width at half maximum. The time course of button-presses in each scan was convolved with a standard model of the haemodynamic response function. For each individual and each scan, blood oxygen level dependent (BOLD) signal fluctuations in all pixels composing a given slice were correlated with the reference time course at temporal shifts ranging from $-4.5 \mathrm{~s}$ to $4.5 \mathrm{~s}$ in 1.5 -s steps, after removing the mean time course across the slice from each pixel time course and from the reference time course. For each time lag and run, correlations were transformed to an approximately normal Gaussian distribution. Gaussian-transformed maps were averaged across runs, yielding a map representing the strength of correlations to the reference button-press time course in terms of standardised $z$-values. The seven $z$-maps of correlations from each participant for each time lag were then transformed to Talairach coordinates. At each Talairach pixel and time lag, a $t$-test was used to assess significance of deviation of $z$-values from zero. This correlation-based analysis can accommodate shorter interhallucination intervals even though the BOLD signal may not return fully to baseline - irregular temporal spacing of hallucinations can improve statistical power, just as 'jittering' allows closer event spacing in event-related designs.

\section{Results}

Positive BOLD signal correlations with hallucination time course were detected selectively at negative time intervals (time lags ranging from $-4.5 \mathrm{~s}$ to $-1.5 \mathrm{~s}$ ) in the left insula and a right middle temporal region, as well as in left pre-central areas (online Fig. DS1). Positive correlations also emerged in the superior temporal gyrus bilaterally at negative time lags, peaking approximately at zero time lag (online Fig. DS1). Negative BOLD signal correlations with hallucination time course were detected in the right ventral anterior cingulate and left parahippocampal gyri at negative time lags.

\section{Discussion}

Two sites exhibiting positive BOLD signal correlations with hallucination time course exclusively at negative time lags largely replicated pre-hallucination activation sites described previously. 


\section{Right middle temporal site}

The right middle temporal site (Brodmann area 21) was located remarkably close to middle temporal gyrus sites of pre-hallucination activation reported by Lennox et al and Shergill et al. ${ }^{1,2}$ Activation in the bilateral superior temporal gyrus (Brodmann area 22) emerged at negative time lags also, but peaked later and was broadly distributed over both positive and negative time lags. Our correlation-based method for mapping BOLD signal time course will produce temporal smearing that broadens with increasing neural activation. The temporal pattern of our data suggests therefore that the more robust bilateral activation in the superior temporal gyrus arose somewhat later - perhaps at hallucination onset - than the middle temporal gyrus activation. Bilateral activation of the latter region has been associated with aspects of verbal comprehension during speech processing distinct from acoustic feature detection referable to the superior temporal gyrus, ${ }^{4}$ whereas non-dominant middle temporal gyral activation has been associated with detecting prosodic features of spoken speech. ${ }^{5}$ One plausible account of our findings is that prehallucination activation in the middle temporal gyrus reflecting verbal content and/or prosody is subsequently propagated to the superior temporal gyrus via top-down processing, which generates (hallucinated) acoustic representations.

\section{Left anterior insula}

The left anterior insula was close to a site of activation in the left inferior frontal gyrus $9 \mathrm{~s}$ prior to hallucination onset identified by Shergill et $a l^{2}{ }^{2}$ who reported expanded activation incorporating the left insula at later times. Left insula activation has been associated with speech articulation, ${ }^{6}$ imagining spoken speech of others, ${ }^{7,8}$ and focused auditory attention, ${ }^{4}$ suggesting that prehallucination insula activation reflects inner speech or auditory imagery generation as previously hypothesised, ${ }^{7,8}$ or enhanced auditory attention. However, pre-hallucination insula activation might instead reflect motor movement required to signal these events. This possibility is suggested by the fact that simple generation of finger movements is preceded by activation in the adjacent Broca's area, which has been postulated to reflect mental preparation. ${ }^{9}$

\section{Other sites}

Evidence of right ventral anterior cingulate and left parahippocampal deactivation preceding hallucination onset was detected. Co-occurring deactivations in these regions have also been linked to heightened vigilance/attention, ${ }^{10}$ suggesting a shift in cognitive state preceding auditory/verbal hallucinations. Along these lines, Arieti described a 'listening attitude' that predisposes people with schizophrenia to hear 'voices. ${ }^{11}$ Pre-central activation emerging prior to hallucination onset in our study could reflect either inner speech generation or signalling hallucinations by finger movement.

In summary, activation detected as BOLD signal changes correlated with auditory/verbal hallucination time course at negative time lags may reveal complex brain processes triggering these experiences. Future studies of this type would be advanced by controlling for effects of motor behaviour required to signal hallucination occurrences. ${ }^{9}$

Ralph E. Hoffman, MD, Department of Psychiatry, Yale University School of Medicine, New Haven, Connecticut; Adam W. Anderson, PhD, Department of Biomedical Engineering and Department of Radiology and Radiological Sciences, Biomedical Engineering and Department of Radiology and Radiological Sciences,
Vanderbilt University, Nashville, Tennessee; Maxine Varanko, PsyD, Department of Psychiatry, Yale University School of Medicine, New Haven, Connecticut; John C. Gore, PhD, Department of Biomedical Engineering and Department of Radiology and Radiological Sciences, Vanderbilt University, Nashville, Tennessee; Michelle Hampson, PhD, Department of Diagnostic Radiology, Yale University School of Medicine, New Haven, Connecticut, USA

Correspondence: Dr Ralph Hoffman, Yale-New Haven Psychiatric Hospital 184 Liberty Street LV108, New Haven, CT 06519, USA. Email: ralph.hoffman@yale.edu

First received 17 May 2007, final revision 28 Sep 2007, accepted 20 Nov 2007

\section{Acknowledgements}

This study was funded by the NIMH grant R01MH067073 (R.E.H.), the Dana Foundation (R.E.H.) and NIH/NCRR/GCRC program grant RR00125.

\section{References}

1 Lennox BR, Bert S, Park G, Jones PB, Morris PG. Spatial temporal mapping of neural activity associated with auditory hallucinations. Lancet 1999; 353 : 644.

2 Shergill SS, Brammer MJ, Amaro E, Williams SCR, Murray RM, McGuire PK. Temporal course of auditory hallucinations. Br J Psychiatry 2004; 185: 516-7.

3 American Psychiatric Association. Diagnostic and Statistical Manual of Mental Disorders (4th edn) (DSM-IV). APA, 1994

4 Giraud AL, Kell C, Thierfelder C, Sterzer P, Russ MO, Preibisch C, Kleinschmidt A. Contributions of sensory input, auditory search and verba comprehension to cortical activity during speech processing. Cereb Cortex 2004; 14: 247-55.

5 Ethofer T, Silke A, Erb M, Herbert C, Wiethoff S, Kissler J, Grodd W, Wildgruber D. Cerebral pathways in processing of affective prosody: a dynamic causal modeling study. Neuroimage 2006; 30: 580-7.

6 Wise RJ, Greene J, Buchel C, Scott SK. Brain regions involved in articulation. Lancet 1999; 353: 1057-61.

7 McGuire PK, Silbersweig DA, Murray RM, David AS, Frackowiak RS, Frith CD. Functional anatomy of inner speech and auditory verbal imagery. Psychol Med 1996; 26: 29-38.

8 Shergill SS, Bullmore ET, Brammer MJ, Williams SC, Murray RM, McGuire PK. A functional study of auditory verbal imagery. Psychol Med 2001; 31: 241-53.

9 Hunter MD, Spence SA. Left frontal activation. Br J Psychiatry 2005; 187: 89

10 Lawrence NS, Ross TJ, Hoffmann R, Garavan H, Stein EA. Multiple neuronal networks mediate sustained attention. J Cogn Neurosci 2004; 15: 1028-38.

11 Arieti S. Interpretation of Schizophrenia. Basic Books, 1994. 